\title{
THE ENERGY SPECTRUM OF SINGLE-HOLE STATES IN TRANSITION METAL OXIDES
}

\author{
A. Lehmann-Szweykowska ${ }^{a}$, R.J. Wojciechowski ${ }^{a}$ AND G.A. GeHring ${ }^{b}$ \\ ${ }^{a}$ Institute of Physics, A. Mickiewicz Univeresity, Umultowska 85, 61-614 Poznań, Poland \\ ${ }^{b}$ Department of Physics and Astronomy, University of Sheffield, Sheffield S3 7RH, U.K.
}

The $p-d$ hybridised single-hole states of the transition-metal-oxygen tetrahedron $\left(\mathrm{TMO}_{4}\right)$ are collectivised due to the direct $p-p$ hopping between oxygens of different clusters. The lowest-lying energy band is always narrow and fully occupied. The first excited band gets occupied as an effect of valence-uncompensated doping, so it can be almost localised. The possible hole excitations to the two higher energy bands, which are wider, may imply the Mott-like hopping form of charge transport in these systems.

PACS numbers: 71.10.Fd, 72.20.-i

\section{Introduction}

Results of the space symmetry analysis support the idea that the basic structural building units of complex oxides are $\mathrm{MO}_{4}$ metal-oxygen tetrahedra and/or $\mathrm{MO}_{6}$ octahedra [1]. The crystal is then considered as constructed of these simple structural elements which can be either mutually-separated, or form corner-, face-, and/or edge-sharing connections $[2,3]$. The $M$ cations are in most cases transition-metal (TM) trivalent ions. The A cations are mostly rare-earth (RE) trivalent cations and the oxygens $(\mathrm{O})$ - divalent. The examples of such materials are garnet-structured $\mathrm{A}_{3} \mathrm{M}_{5} \mathrm{O}_{12}$ and $\mathrm{AMO}_{3}$ perovskite-structured oxides. Charge carriers are created mainly by valence-uncompensated doping, however, they can be also introduced by annealing treatments, or by light excitation techniques. In this paper, we present an approach which is intended as a first step towards an understanding of the development of charge conductivity in such systems.

The model we propose, is a simple cubic lattice consisting of $\mathrm{TMO}_{4}$ tetrahedra, separated from one another. The electronic structure of the system is described in terms of compensating holes which fall into two categories: $d$ holes localised at the TM cations and $2 p$ holes localised at the oxygen sites. The cubic crystal field splits 5 orbital states of each $d$ hole into the $t_{2 g}$ triplet, being the ground state at a tetrahedral site, and the excited doublet $e_{2 g}$. The $2 p$ orbital states at an oxygen site form the triplet $t_{1 u}$ (see: for example, [4]). The $d$ holes are treated in the local (atomic) limit of the standard two-band Hubbard Hamiltonian with the correlation between holes of the opposite spins, and the cubic crystal field. At the oxygen 
sites, the opposite-spin Hubbard correlations are neglected, but the Hamiltonian is extended to include the direct $p-p$ hopping. Finally, the $p-d$ hybridization is introduced connecting the two subsystems of the holes with each other.

\section{The single-hole states of the $\mathrm{MO}_{4}$ tetrahedron}

The first step in our approach is a thorough analysis of the $\mathrm{MO}_{4}$ tetrahedral cluster. Within the framework of the linear combination of atomic orbital (LCAO) method, a cluster wave function can be approximated as a linear combination of atomic orbitals attached to the individual sites. The number of possible molecular orbitals is limited to those constructed of the TM and oxygen atomic orbitals between which the $p-d$ hybridization term has non-zero matrix elements. The appropriate atomic orbitals can be precisely determined by the group-theoretical methods [1]. Here, it is only assumed that those are the orbital singlets. The remaining atomic states at each site of the cluster are neglected since they provide no contribution to the hole motion. In the standard perturbation procedure, we arrive at four energy levels and their respective eigenstates constructed of the symmetry-permitted atomic orbitals with the $p-d$ hybridization treated as a perturbation. The $p-d$ hybridization parameter, $V$, is considered as small with respect to the cost of the hole transfer from the $d$ to $2 p$ state, i.e. $\Delta=E_{p}-E_{d}$, and the Hubbard correlation parameter, $U$. Coefficients of the linear combinations of the $d$ and $2 p$ atomic orbitals are expressed in terms of the system parameters $\left(E_{d}, E_{p}, U\right.$, and $\left.V\right)$. The holes at a cluster have a combined $d$ and $2 p$ character. Two of the four cluster states have their spin opposite to that of the others.

\section{The electronic structure of the crystal}

The system is then considered as a quasi-crystal constructed from metaloxygen clusters with the four $p-d$ single-hole states of distinct energies, attached to each of them $[2,3]$. The clusters, labelled by their TM cations, are separated from one another, so the compensating holes get trapped and can only be released by the direct $p-p$ hopping between oxygens belonging to different clusters. The original $p-p$ hopping integral, $t$, is modified by coefficients of the $2 p$ atomic orbital contributions to the cluster hole states. The symmetry analysis allows effective jumps both without and with a change of the hole orbital state. Only the spin is preserved in the process of the effective cluster-to-cluster hopping. Therefore, six effective hopping integrals are found corresponding to four jumps with the hole state preserved and two jumps accompanied by a change of the state. The effective Hamiltonian subjected to the Fourier space transformation and the diagonalization with respect to the spin, provides the following energy bands:

$$
\epsilon_{k, \mu}=(1 / 2)\left[E_{1}+t_{11}(k)+E_{3}+t_{33}(k)\right] \mp(1 / 2) \sqrt{\delta_{k}},
$$

where $\delta_{k}=\left[E_{3}+t_{33}(k)-E_{1}-t_{11}(k)\right]^{2}+4 t_{13}(k) t_{31}(k)$. The value of the spin for the states $\mu=1,3$ is equal to $m_{s}= \pm 1 / 2$. The energies of the localised cluster states with the spin $m_{s}$ are equal to

$$
E_{\mu}=(1 / 2)\left(E_{d}+E_{p}\right) \mp(1 / 2) \sqrt{\Delta^{2}+4 V^{2}} .
$$


Analogous expressions can be obtained for the states with the opposite spin, i.e. $-m_{s}$. The energy bands are as follows:

$$
\epsilon_{k, \mu}=(1 / 2)\left[E_{2}+t_{22}(k)+E_{4}+t_{44}(k)\right] \mp(1 / 2) \sqrt{\delta_{k}} .
$$

In this case, $\delta_{k}=\left[E_{4}+t_{44}(k)-E_{2}-t_{22}(k)\right]^{2}+4 t_{24}(k) t_{42}(k)$, where $\mu=2,4$ correspond to the spin equal to $-m_{s}$. The energies of the localised cluster states with the spin $-m_{s}$ are equal to

$$
E_{\mu}=(1 / 2)\left(E_{d}+U+E_{p}\right) \mp(1 / 2) \sqrt{(U-\Delta)^{2}+4 V^{2}} .
$$

The Fourier transforms of the respective effective hopping integrals are defined in the usual form with the summation over the nearest clusters. The effective intra-state hopping integrals take the following form:

$$
t_{\mu \mu}(k)=\left[(1 / 8) t\left(1+\alpha / \sqrt{1+\gamma^{2}}\right] \sum_{j(n n)} \exp \left(\mathrm{i} k \boldsymbol{R}_{j}\right)\right.
$$

( $\alpha=\mp 1$ for $\mu=1,3$ and $\alpha= \pm 1$ for $\mu=2,4$ ). The effective integrals which correspond to the inter-state hopping, fulfil the following relation: $t_{\mu \mu^{\prime}}(k)=t_{\mu^{\prime} \mu}(k)$. And

$$
t_{\mu \mu^{\prime}}(k)=-(1 / 8) t[1-1 /(1+\gamma)] \sum_{j(n n)} \exp \left(\mathrm{i} k \boldsymbol{R}_{j}\right),
$$

$\gamma=2 V / \Delta$ for $\mu, \mu^{\prime}=1,3$ whereas for $\mu, \mu^{\prime}=2,4 \gamma=2 V /(U-\Delta)$.

The four energy bands (see e.g. Fig. 1) are obtained for different values of the system parameters approximated for TM oxides on the basis of literature on the subject (see: for example [5]). Also, the four band widths are calculated and compared to the localisation energy for a defect state associated with the

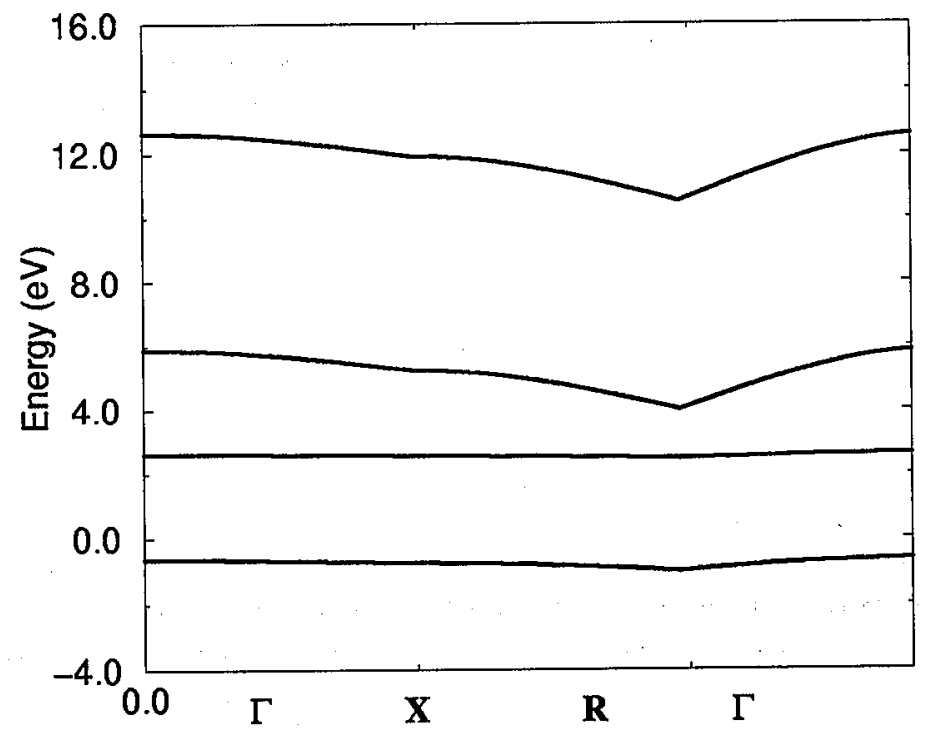

Fig. 1. The band energy in the direction $\Gamma X R \Gamma$ of the simple cubic first Brillouin zone. 
dopant ion. Actual estimation of these values is done for $\mathrm{Ca}^{+2}$-doped yttrium-iron garnet (YIG). The binding energy of holes to the $\mathrm{Ca}$ is obtained from the following simple formula: $E=-e^{2} /\left(8 \pi \epsilon_{0} \epsilon R\right)$, where $R$ is the distance between the calcium and the nearest oxygen and $\epsilon$ is the dielectric constant. The value of the energy is about $-0.2 \mathrm{eV}$ for $\epsilon=15$ which is assumed for YIG [6]. For a certain set of parameters $(U=5 \mathrm{eV}, \Delta=4 \mathrm{eV}, V=2.5 \mathrm{eV}$ and $t=0.3 \mathrm{eV})$ three energy bands $\left(\epsilon_{k, 1}, \epsilon_{k, 2}\right.$, and $\left.\epsilon_{k, 4}\right)$ have their widths equal to $0.10 \mathrm{eV}, 0.23 \mathrm{eV}$, and $0.19 \mathrm{eV}$, respectively. They are rather narrow, so localised states are allowed. No charge transport is possible unless some holes get excited from the first band to the next band with the same spin direction $\left(\epsilon_{k, 3}\right)$ and its width is equal to $0.32 \mathrm{eV}$. On the whole, the band widths turn out to be very sensitive to all the system parameters and especially to the oxygen-oxygen hopping. Its parameter $(t)$ has been varied in a rather wide range and, for instance, if $t=0.3 \mathrm{eV}$ the width of the narrowest band is equal to $0.1 \mathrm{eV}$ and the localisation certainly occurs.

\section{Summary}

Let us summarize the obtained conclusions. The localised combined $p-d$ hole states of each metal-oxygen tetrahedral cluster are obtained within the framework of quantum-chemical standard LCAO approach. The localised states are then collectivised due to the $p-p$ inter-cluster hopping, giving rise to four bands of different widths. Thus the hole motion in the crystal is mediated both by the $p-d$ hybridization and direct $p-p$ hopping, and moreover, it can be accomplished after excitations to higher lying bands which contribute in explanation of the thermally activated hopping character of the electric conductivity of YIG $[6,7]$.

The authors (A.L.S. and R.J.W.) acknowledge support from the Committee for Scientific Research (Poland) under grant No. 2 P03B 05614.

\section{References}

[1] M.M. Kaczmarek, Ph.D. Thesis, Adam Mickiewicz University, Poznan 1998.

[2] H. Donnenberg, S. Többen, A. Birkholz, J. Phys., Condens. Matter 9, 6359 (1997).

[3] A.J. Millis, Phys. Rev. B 55, 6405 (1997).

[4] L.F. Feiner, A.M. Oleś, Phys. Rev. B 59, 3295 (1999).

[5] G.A. Sawatzky, in: Earlier and Recent Aspects of Superconductivity, in Springer Series Solid-State Sciences, Vol. 90, Eds. J.G. Bednorz, K.A. Müller, Springer-Verlag, Berlin 1990, p. 345.

[6] Y.J. Song, Ph.D. Thesis, The Ohio State University, Ohio 1993, ch. V.

[7] M. Pardavi-Horvath, S.H. Yuan, P.E. Wigen, P. DeGasperis, J. Magn. Magn. Mater. 119, 205 (1993). 